\title{
Téoros
}

Revue de recherche en tourisme

\section{Le marketing du tourisme d'aventure : un quadruple défi stratégique}

\section{Renée Dubé et Michel Zins}

Volume 13, numéro 3, automne 1994

Le tourisme d'aventure : vers la maturité ?

URI : https://id.erudit.org/iderudit/1077112ar

DOI : https://doi.org/10.7202/1077112ar

Aller au sommaire du numéro

Éditeur(s)

Université du Québec à Montréal

ISSN

0712-8657 (imprimé)

1923-2705 (numérique)

Découvrir la revue

Citer cet article

Dubé, R. \& Zins, M. (1994). Le marketing du tourisme d'aventure : un quadruple défi stratégique. Téoros, 13(3), 38-39. https://doi.org/10.7202/1077112ar d'utilisation que vous pouvez consulter en ligne.

https://apropos.erudit.org/fr/usagers/politique-dutilisation/ 


\title{
Le marketing du tourisme d'aventure : un quadruple défi stratégique
}

\author{
Renée Dubé et Michel Zins*
}

Le marketing d'un produit ou d'une entreprise touristique qui chercherait a tirer profit de l'engouement encore relativement récent et toujours croissant pour le tourisme d'aventure constitue un quadruple défi :

- identifier et choisir les clientèles à cibler;

- se positionner et définir les avantages concurrentiels;

- s'assurer de l'accessibilité et de la livraison adéquate du produit promis;

- rejoindre, convaincre et bien informer les dientèles visées.

Ce quadruple défi s'inscrit dans un contexte caractérisé par ${ }^{(1)}$ :

- un concept de tourisme d'aventure qui a évolué vers une définition beaucoup plus large qu'auparavant, alors qu'il se limitait à une expérience presqu'inaccessible à l'individu moyen;

- des grossistes en voyages de plein air et de villégiature qui offrent des produits plus variés;

- une industrie du tourismeet desvoyageurs qui sont affectés parl'émergence et, récemment, l'explosion de la demande pour un tourisme plus environnementaliste;

- une confusion grandissante entre les concepts mal définis de tourismevert, d'écotourisme, de tourisme d'aventure, de voyages orientés vers la nature et des voyages motivés par la pratique des activités de plein air.

\section{L'enjeu des clientèles}

La clientèle du tourisme d'aventure a beaucoup évolué et ses perspectives de croissance sont très grandes. Mais elle a connu et connaîtra encore des mutations importantes. En effet, le touriste amateur d'aventure d'origine (l'aventurier pur et dur) n’a pas disparu, mais il a été supplanté ou talonné par une cohorte de plus en plus grande de touristes recherchant différentes expériences reliées à l'aventure. Ceuxci ne veulent toutefois pas renoncer à la

Madame Renée Dubé est directrice chez Zins Beauchesne et associés et monsieur Michel Zins es1 president chez Zins Beauchesne et associés. sécurité et à un minimum d'hygiêne et de confort, ou prendre les mêmes risques que l'aventurier pur et dur. Cette clientèle est aussi moins apte à fournir les efforts physiques requis par les expéditions du tourisme d'aventure de la première heute.

Les motivations du touristed'aventure sont variéeset, suivantles individus, on retrouve un accent plus ou moins grand sur certains des bénéfices (tableau 1).

Mais les clientèles varient également en fonction d'un certain nombre de caractéristiques:

- forme physique;

- niveau d'habileté;

- expériences antérieures pertinentes;

- volonté (ou capacité) de payer;

- disponibilité (facteur temps);

- sćcurité (éviter les risques).

De plus, le tourisme d'aventure, tout en ayant peut-être été à l'origine du développement de l'écotourisme et du tourisme vert, etpeut-êtremême del'ethnotourisme, est en voie d'être rattrapé et englouti, voire marginalisé par ces dernières formes de tourisme. L'écotourisme et le tourisme vert s'adressent, avec des produits adaptés, à une clientèle cousine de celle du tourisme d'aventure, quoique beaucoup plus nombreuse et craintive face au risque et à l'effort.

Le défi pour le promoteur de tourisme d'aventure est de bien définir les aspirations et les motivations des clientèles a qui il veut offrir son produit.

En effet, toutes les clientèles ne se marient pas forcément à l'intérieur d'un même groupe ou sur un même territoire. Le voyageur contemplatif sera excédé par l'aventurier athlétique, etréciproquement. De même, l'aventurier solitaire sera irrité par les efforts déployés par l'aventurier social qui cherche une expérience de vie commune.

Il faut donc retenir que l'on ne peut servir toutes les clientêles en même temps. Il faut segmenter le matché, choisir lesclients que l'on veut servir, etce, efficacement et à leur entière satisfaction. Le compromis entre diverses clientèles (voire les conflits entre celles-ci) sera mortel pour l'aventure.

\section{Positionnement et avantages concurrentiels}

Le choix des clientèles visées, s'il doit être fait en fonction du potentiel de marché, doit également être déterminé en fonction du positionnement et des avantages concurrentiels quele promoteur veut mettre de l'avant.

Le producteur de tourisme d'aventure doit se pasitionnersur unatuntage concurrentiel, c'età-dire une supériorité sur sa concurrence, par rapport à un élement jugé de prenière importance par la clientèle qu'il vise.

Ainsi, on peut noter que différents producteurs vont mettre l'accent dans leur offre sur des éléments différents :

- activités(trekking, rafting, canoë, etc);

- régions géographiques (Galapagos, Kenya, Amazonie, Alaska, Sahara, Groenland, Anticosti, Torngat, etc.);

- attraits naturels ou points d'intérêts (Grand Canyon, Anapurna, Yellowstone, chutes Victoria, Archipel de Mingan, etc.);

- gites de montagne, camps, ranchs, stations grande nature;

- expérience didactique (biologie, géologie, volcans, flore et faune, etc.);

- observation de la nature (ours polaire, morse, cheetah, etc);

- défis (escalader le Kilimandjaro, descendre la rivière Amazone, traverser le désert du Sahara, survivre à l'hiver arctique, etc.).

L'essentiel reste que l'offre revête un caractère d'unicité sur un aspect important pour la clientèle visée, les autres aspects devant au moins demeurer comparables en regard de la concurrence.

Cettesupériorité par l'unicité peutse rattacher à la destination ou à l'expérience ellemême, à l'encadrement ou aux services de soutien offerts. Elle peut parfois également se rattacher à la durée (courte ou longue), à la taille des groupes (limitée), au mode d'hébergement ou de transport, à la saison du voyage, ou même au prix. 
TABLEAU 1 : BENEFICES

\begin{tabular}{|c|c|}
\hline Physiques & $\begin{array}{l}\text { - reliés à une expérience sensorielle; } \\
\text { - suite à un effort physique, } \\
\text { - par un effet positif sur la santé. }\end{array}$ \\
\hline Apprentissage et compréhension de & $\begin{array}{l}\text { l'environnement; } \\
\text {. la geologie; } \\
\text {. la faune; } \\
\text {. la flore; } \\
\text {. la culture; } \\
\text { l'histoire; } \\
\text { - etc. }\end{array}$ \\
\hline Acquisition d'habiletés & $\begin{array}{l}\text { - escalade; } \\
\text { - cyclisme; } \\
\text { - etc. }\end{array}$ \\
\hline Émotions et sensations & $\begin{array}{l}\text {. excitation; } \\
\text { - commémoration; } \\
\text { - spiritualité; } \\
\text { - reve; } \\
\text {, contemplation. }\end{array}$ \\
\hline Aspect social & $\begin{array}{l}\text { prestige; } \\
\text { - amitié (créer des liens); } \\
\text { - vie de groupe; } \\
\text { - vie familiale. }\end{array}$ \\
\hline
\end{tabular}

\section{L'accessibilité sans les foules}

La croissance et la mutation du marché du tourisme d'aventure ont entraîné une exigence particulière d'accessibilité pour les promoteurs de tourisme d'aventure. On s"éloigne de plus en plus de l'aventurier capable et désireux de s'organiser tout seul et qui n'a besoin de rien. Du point de vue de l'industrie, ce dernier touriste n'est pas forcément le plus intéressant.

De plus en plus de personnes plutôt ordinaires, qui veulent vivre une aventure dont elles rêvent depuis longtemps ou qu'elles ont idéalisée, viennent frapper à la porte desopérateursde tourismed'aventure. Ces personnes n'ont pas nécessairement le temps ou les connaissances pour organiser un tel voyage.

Mais comment rendre accessible un produit d'aventure aux bonnes clientèles? Rendre le produit du tourisme d'aventure accessible représente un dilemme: c'est justement l'inaccessibilité perçue des lieux et des sites ${ }^{(1)}$ qui en fait son attrait. Rendre ces destinations trop accessibles pourrait non seulement avoir un impact néfaste sur l'environnement, la faune et la flore, mais aussi sur la perception des clientèles.

A titre d'exemple, l'observation des baleines en zodiac reste un produit d'aventure ou écotouristique, alors que les croisières sur des bateaux luxueux de 300 personnes n'ont plus rien à voir avec l'aventure. Une trop grande concentration de ce genre de tourisme pourrait ainsi faire fuir la clientèle d'aventure ou écotouristique, au grand détriment des baleines et de l'environnement.

Or, si l'on doit limiter l'accessibilité plysique au tourisme d'aventure, l'accéssibilité en termes de servicé doit cependant être préservée ou accrue. Pour les opérateurs, ceci signifie qu'ils doivent être capables de fournir une expérience agréable, enrichissante et stimulante dont le client sera satisfait à partir du moment de sa décision devoyager jusqu'à son retour. En pratique, il s'agit :

- de bien servir, informer et renseigner le client sur les produits offerts (la section suivante explique comment);

- de s'occuper de tous les aspects techniquesqui peuventdevenir des irritants pour les clients (réservations, transferts, équipements, assurances);

- de s'assurer de l'accueil à destination et de la qualité du service offert;

- d'informer le client sur les trajets, routes et déplacements à faire, et ce que cela implique comme niveaux d'efforts, d'habiletéset desavoir-faire, comme type d'équipement;

- d'assurer ou organiser le transport (aérien, terrestre, maritime) pour se rendre au point de départ du circuit d'aventure;

- d'introduireune mesure de la satisfaction du client a posteriori et de corriger certaines actions s'il $y$ a lieu.
Rejoindre, convaincre et bien informer les clientèles visées

Les tentatives pour rejoindre les clientèles en les ciblant sur la base de critères socioéconomiques et démographiques (revenu, âge, sexe, etc.) ont perdu leur pertinence dans les années 1990. Aujourd'hui, «tout le monde fait du tourismed'aventure». Ainsi, une segmentation sur la base de critères psychologiques, tels l'intérêt pour certains types d'activités ou les bénéfices recherchés, s'avère dorénavant plus utile pour cibler lesclientèles. Lesopérateurs peuvent mettre en oeuvre différents moyens pour rejoindre la bonne clientèle :

- publier des annonces dans les revues spécialisées dont le sujet d'intérêt est le même ou proche de celui du produit d'aventure;

- passer par un grossiste vendant des produits de nature similaire (ex: rafting), de même intérêt (ex,: ornithologie) ou procurant les mêmes bénéfices (ex.: dépassement de soi);

- avoir son propre catalogue (ex.: Club Aventure, opérateur et promoteur);

- lancer des opérations de marketing direct (envois ciblés, télémarketing à partir de listes de clients potentiels, etc), techniquesouvent employée par les plus petits opérateurs mais qui peut devenir très coûteuse à grande échelle. Cette méthode s'avère par contre très efficace pour offrir le bon produit aux bonnes clientèles;

- utiliser des rédactionnels dans les revues spécialisées ou d'intérêt plus génếral.

\section{Conclusion}

Le défi des opérateurs de tourisme d'aventure est donc simple :

- avoir et livrer un bon produit...

- qui se distingue de la concurrence par des avantages différentiels réels...

- accessible, mais pas trop, aux clientèles recherchées..+

- que l'on rejoint et que l'on informe par des moyens permettant de les abler et de répondre à leurs attentes.

\section{NOTES}

(1) Renée Dubế, M.Sc Adventure Travel Redefined: Product Differentiation and Segmentation of Clients and Markets, TTRA Canada and New England Joint Conference, 1993.

(2) Fienée Dubé, lbid.

(3) Inaccessibilité scit par l'éloignement des lieux, th limitation du nombre de personnes par groupe ou le niveau de difficulté ou de risque pour s'y rendre. 